\title{
Future of the CMS Muon System: Upgrades and Aging
}

\author{
Justin Pilot ${ }^{* \dagger}$ \\ University of California Davis (US) \\ E-mail: justin.pilotecern.ch
}

The CMS detector currently includes three different muon detector types: drift tubes (DT) in the central region, cathode strip chambers (CSC) in the forward regions, and resistive plate chambers (RPC) in both the forward and central regions. Several upgrade projects are planned to maintain high data-taking efficiency with the planned running conditions for the high-luminosity upgrade of the LHC. These upgrades are designed to ensure detector longevity and increase redundancy, while mitigating rate increases and retaining sensitivity to phyics processes. This involves changes to electronics and infrastructure of existing detectors, and adding new detectors in the forward region of the CMS experiment. Plans for each of the muon subsystems are described here in the context of the Phase-II upgrade schedule of the CMS experiment.

38th International Conference on High Energy Physics

3-10 August 2016

Chicago, USA

${ }^{*}$ Speaker.
†On behalf of the CMS Collaboration 


\section{Introduction}

Over the course of 2016, the CERN LHC and its experiments have been performing incredibly well. The LHC has reached record instantaneous luminosities of above $1.3 \times 10^{34} \mathrm{~cm}^{-2} \mathrm{~s}^{-1}$, while the experiments have maintained high data-taking efficiencies. The datasets analyzed for ICHEP 2016 analyses correspond to more than $12 \mathrm{fb}^{-1}$ of integrated luminosity, a testament to the performance of both the detector and the accelerator teams.

We have had the last major increase in collision energy for some time, when the LHC moved from $\sqrt{s}=8 \mathrm{TeV}$ to $13 \mathrm{TeV}$. However, the instantaneous luminosity is expected to increase further by a factor of $\sim 5$ in the future, up to $5-7 \times 10^{34} \mathrm{~cm}^{-2} \mathrm{~s}^{-1}$. This upgrade of the CERN LHC complex, known as the 'high-luminosity LHC' (HL-LHC), will first start with upgrades to the injectors and collimators in 2019-2020, followed by the full completion of the project in 20242026.

The HL-LHC proposal will provide challenging running conditions for the experiments. With the expected instantaneous luminosities, as many as 140 additional interactions (pileup) per bunch crossing are expected. This leads to higher background rates, especially in the forward region. This document will summarize the plans for the CMS muon systems in the context of the HL-LHC upgrade plan. Several upgrades are planned to ensure detector longevity and data-taking efficiency, and new detectors are anticipated to be installed to increase redundancy and geometrical coverage of the interactions. Together, the upgrade projects for all detector components in preparation for the HL-LHC are known as the 'Phase-II' upgrade of the CMS experiment [1].

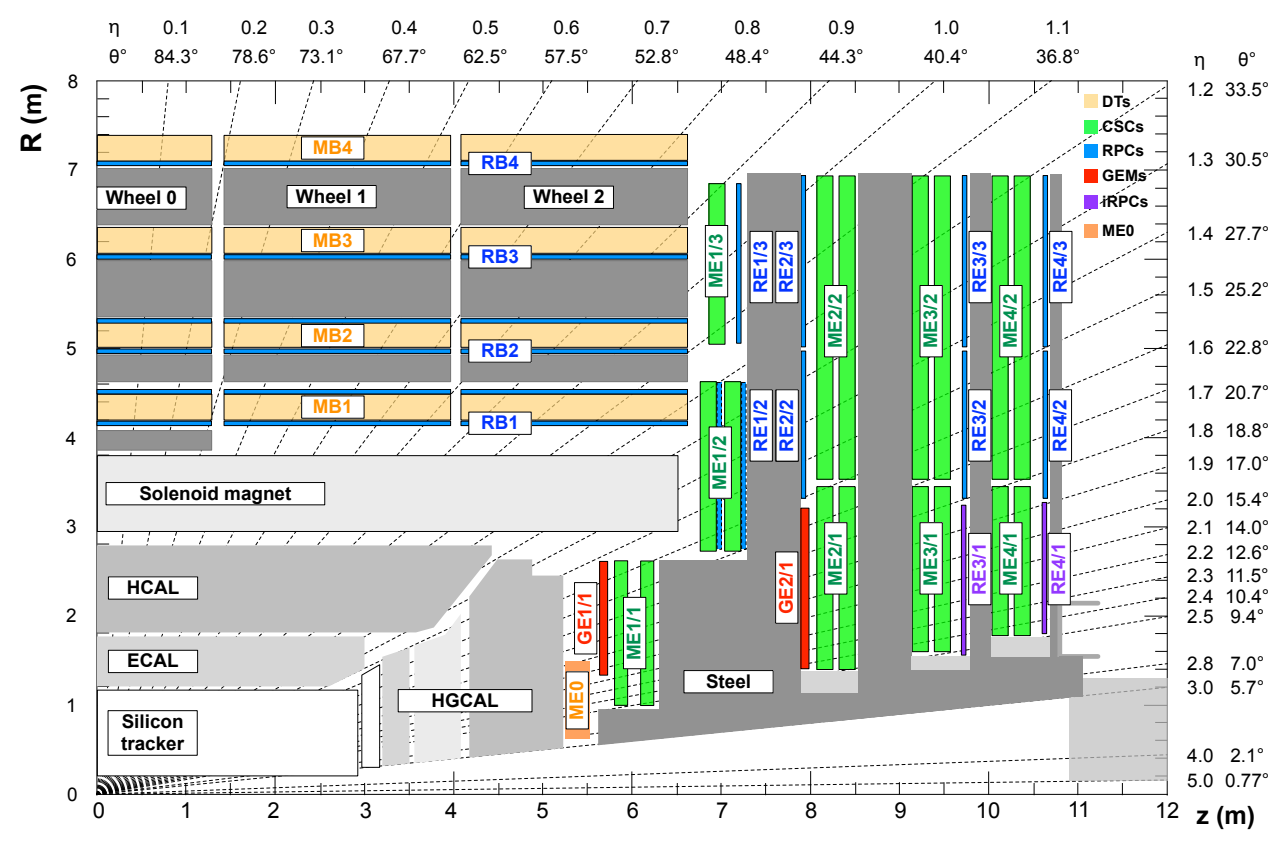

Figure 1: Diagram of one quadrant of the future CMS detector after the completion of the phase II upgrades. The existing muon systems are shown in yellow (DT), blue (RPC), and green (CSC), while the planned new detectors in red (GEM), orange (forward muon tagger), and purple (improved RPC). Other elements of the CMS detector are shown in gray. 


\section{CMS Muon Systems}

The CMS muon system consists of three separate subdetectors: the drift tubes (DT), the resistive plate chambers (RPC), and the cathode strip chambers (CSC). Figure 1 shows these subdetectors in the context of the entire CMS detector. The DT detectors are located in the central region of the detector and extend to $|\eta|<1.2$. The RPC detectors are interspersed with the DT and CSC elements, and have geometrical coverage of $|\eta|<1$.6. Finally, the CSC detectors extend the muon coverage with four layers covering $0.9<|\eta|<2.4$.

Figure 1 also shows the proposed new detectors for the Phase II upgrade, including the gas electron multiplier (GEM) detectors, improved RPC detectors, and the forward muon tagger known as ME0. These proposed new detectors will be detailed in a following section.

\section{Phase II Upgrade Strategy}

The strategy for CMS during the Phase II Upgrade can be divided into four main areas: ensuring detector longevity, increasing redundancy, mitigating rate increases, and retaining sensitivity to physics processes. Detector longevity can be ensured by changes to readout electronics and associated equipment. In the region $1.5<|\eta|<2.4$, currently only the CSC subsystem has active detector layers. Figure 2 shows the number of muon detector layers as a function of pseudorapidity $\eta$, along with the expected neutron flux for the HL-LHC. The detector region with the lowest number of active layers overlaps exactly with the region of high neutron background. Here, it can be beneficial to add additional detector coverage. To mitigate rate increases, new high-rate detectors are proposed to discriminate against backgrounds, and new trigger strategies are planned to maintain reasonable thresholds for physics events. In this section we detail these strategies for each of the existing muon subdetectors, then describe plans for new detectors to be installed for Phase II.

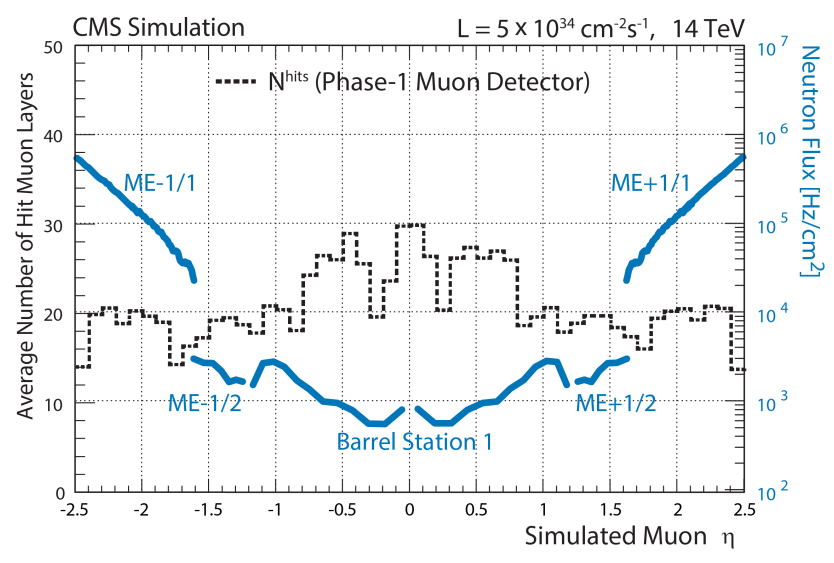

Figure 2: The average number of hit muon layers in the CMS detector (all subsystems) as a function of the muon pseudorapidity $\eta$, shown in the black dashed line. The superimposed blue line shows the expected neutron flux for HL-LHC luminosities. 


\subsection{Existing Detectors}

Several changes to the existing muon detectors are envisioned for the Phase II upgrade, to perform efficiently with the expected HL-LHC running conditions.

The majority of the current front-end electronics for the CSC detectors rely on analog technology to transfer data from the detector to the CMS trigger and reconstruction systems. The HL-LHC conditions and trigger rates of up to $750 \mathrm{kHz}$ will cause these analog pipelines to saturate, causing a significant amount of data loss. The fraction of events lost to this problem is expected to be about $5 \%$ for the outer layers, but even higher for the innermost layers of the subdetector.

To avoid this data loss, an electronics upgrade is proposed for the most forward chambers of the CSC system, the ME2/1, ME3/1, and ME4/1 layers as shown in Figure 1. The readout electronics boards will be replaced by digital equivalents with improved memory depth and fast optical links for data transfer. This will reduce the event loss fraction to a negligible level for the expected latencies and rates. A similar upgrade was done for the ME1/1 layer during the long shutdown of the LHC in 2012-2014; this upgrade would use similar readout electronics.

Aging studies have been performed for the DT detectors, to estimate the performance after integrating $3000 \mathrm{fb}^{-1}$ at HL-LHC luminosities. The DT detectors are expected to operate through those conditions with a conservative estimate of $6.3 \%$ efficiency lost. The loss could be due to several factors, including electronics or high voltage failures, as well as lower efficiency due to the integrated dose of radiation. The efficiency loss could be further mitigated by, for example, adjusting high voltage settings of the detectors as the instantaneous luminosities change.

Similar to the CSC system, changes to the DT electronics are also needed to maintain performance of the readout chain. Part of this chain will be moved away from the detector itself using fast gigabit optical links. The changes to the electronics will also improve the timing resolution of the detector from $12.5 \mathrm{~ns}$ down to $3 \mathrm{~ns}$, allowing a factor of 4 reduction in deadtime.

The current RPC detectors, in use in both the barrel and endcap regions of CMS, are projected to be able to cope with the rates expected from the HL-LHC running conditions. Additional 'improved RPC' detectors are proposed to restore the redundancy in the forward region $1.6<|\eta|<2.4$. These detectors are described in the following section.

\subsection{Proposed New Detectors}

In addition to the electronics and associated changes to the existing muon detectors, several new detectors are proposed in the forward region of CMS. These new detectors can be seen in the CMS schematic shown in Figure 1.

First, two layers of gas electron multiplier (GEM) detectors will be installed in front of the existing CSC chambers [2], covering the range $1.6<|\eta|<2.2$. The GE1/1 layer will be installed in 2018-2019 (LHC long shutdown 2) while the GE2/1 layer is scheduled for LHC long shutdown 3 in 2023-2025. The signals from these chambers will be integrated with the CSC trigger signals, where the bending angle between the CSC and GEM detectors can be used for discrimination. The addition of the innermost layer, GE1/1, will reduce the trigger rate by a factor of $2-4$ or more, depending on the muon $p_{T}$. This means that trigger thresholds will not have to be compromised in order to maintain a high sensitivity to physics signals. Figure 3 shows a comparison of the trigger rates with and without the GE1/1 layer, showing this expected rate reduction. 


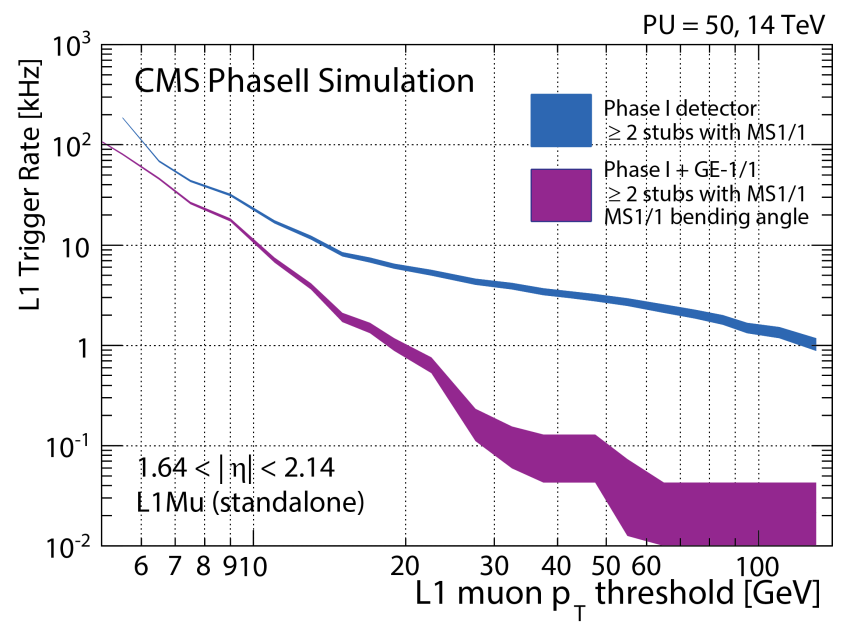

Figure 3: Expected trigger rates before and after the installation of the GE1/1 layer as a function of the muon $p_{T}$ threshold. The blue curve shows the current detector where only the ME1/1 layer is used. The violet curve shows the expected rate after the installation of GE1/1, with the added use of the bending angle between ME1/1 and GE1/1.

As mentioned previously, the RPC detectors are currently in use in both the barrel and endcap regions of CMS. For the Phase II upgrade, improved RPC (iRPC) chambers are to be added to restore the redundancy in the forward region of $1.6<|\eta|<2.4$. These new detectors have an improved design and need to be compatible with the especially high rates expected in the forward region of the detector. These iRPC detectors can perform with particle rates up to $2 \mathrm{kHz} / \mathrm{cm}^{2}$, and have a possible option for a timing resolution of as low as $100 \mathrm{ps}$. These factors together help to mitigate rate increases due to neutron backgrounds. Figure 4 shows the gain in efficiency obtained for the ME3/1 layer before and after the addition of the new iRPC detectors in layer RE3/1.

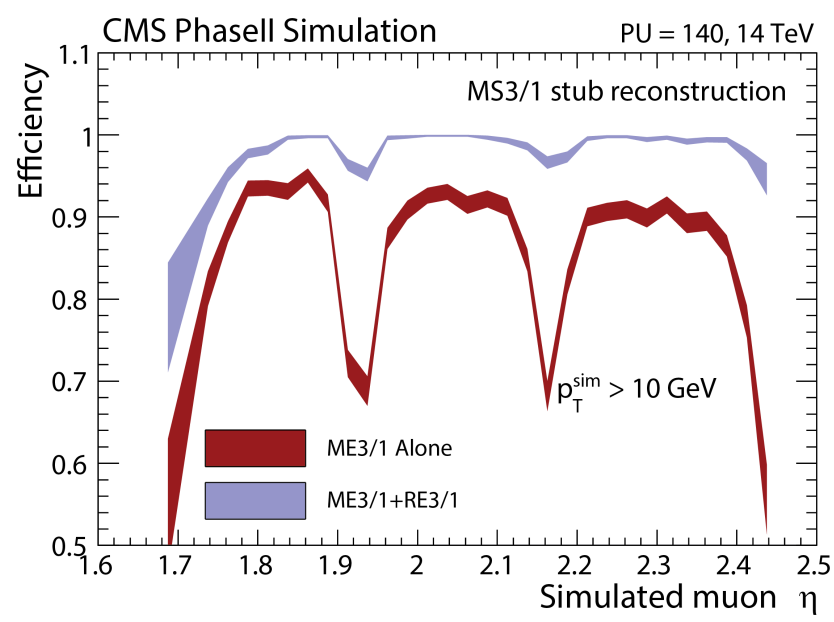

Figure 4: Muon stub reconstruction efficiency as a function of muon $\eta$, for simulated muons with $p_{T}>10$ $\mathrm{GeV}$. The red line shows the efficiency for the ME3/1 layer alone, while the blue line shows the improved efficiency after the addition of the RE3/1 layer. 
Finally, with the new forward calorimetry planned for CMS, some space is freed in the far forward region for further instrumentation. Additional GEM chambers with associated neutron shielding are planned to increase the muon system coverage to $|\eta|<2.8$. These new chambers can improve the trigger coverage for muons, as well as discriminate against the high neutron background in the forward region. Studies show a muon identification efficiency of about $90 \%$ can be obtained out to $\eta= \pm 2.8$, for $p_{T}>5 \mathrm{GeV}$.

\section{Summary}

The CMS experiment has several plans to upgrade and add to the muon system for the Phase II program. These plans involve changes to the electronics and readout chains of existing detectors, as well as the installation of new high-rate, radiation-hard detectors. Figure 5 shows the timeline for design, construction, installation, and commissioning of these detectors in preparation for HLLHC running. These upgrades will allow CMS to maintain highly efficient operation through the HL-LHC era.

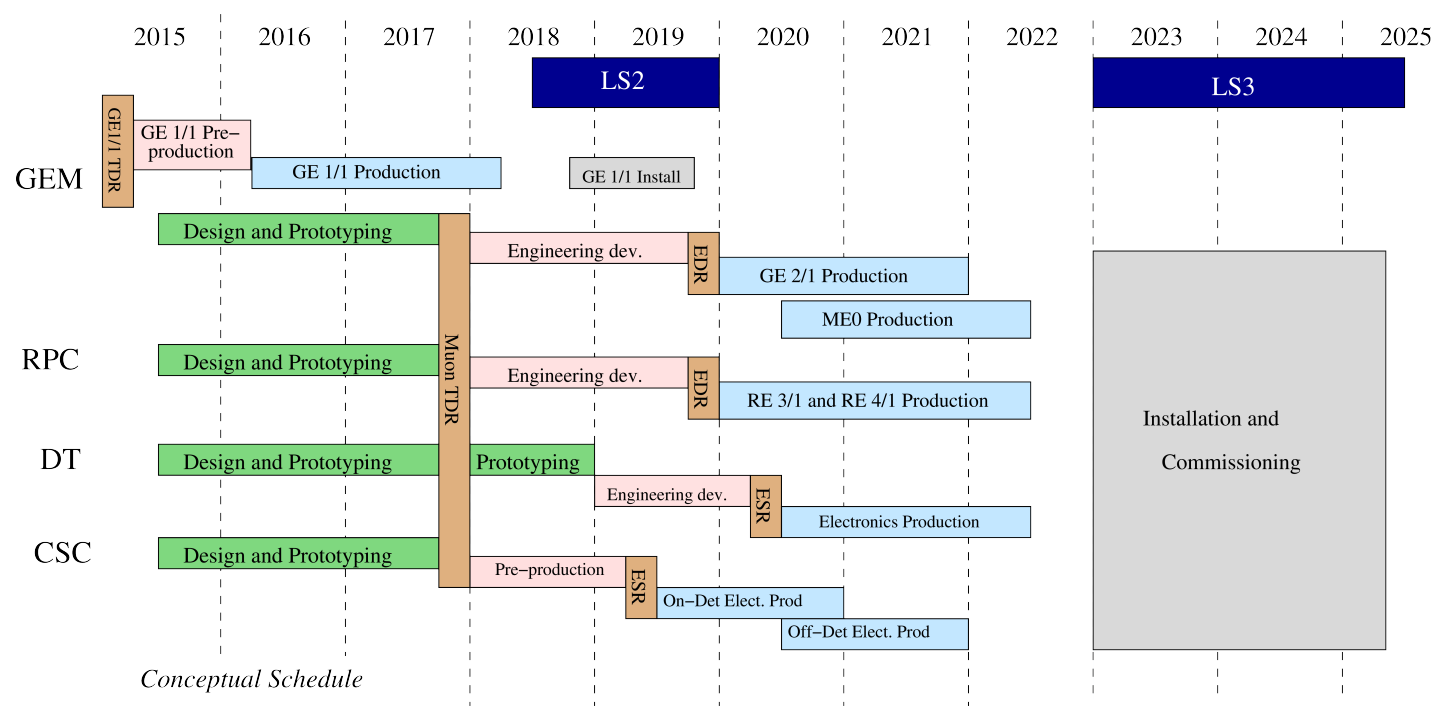

Figure 5: Conceptual schedule for the Phase II upgrades planned for each of the muon subsystems.

\section{References}

[1] CMS Collaboration, "Technical Proposal for the Phase-II Upgrade of the CMS Detector" (2015), CMS-TDR-15-002, LHCC-2015-010, cds . cern . ch/record/2020886

[2] CMS Collaboration, "CMS Technical Design Report for the Muon Endcap GEM Upgrade" (2015), CMS-TDR-013, LHCC-2015-012, cds . cern. ch/record/2021453 